\title{
Measurements and analysis of acoustic backscattering by elastic cubes and irregular polyhedra
}

Thorne, P D; Sun, S; Zhang, J; Bjørnø, Irina; Mazoyer, T

\section{Published in:}

Acoustical Society of America. Journal

Link to article, DOI:

10.1121/1.420324

Publication date:

1997

Document Version

Publisher's PDF, also known as Version of record

Link back to DTU Orbit

Citation (APA):

Thorne, P. D., Sun, S., Zhang, J., Bjørnø, I., \& Mazoyer, T. (1997). Measurements and analysis of acoustic backscattering by elastic cubes and irregular polyhedra. Acoustical Society of America. Journal, 102(5), 27052713. https://doi.org/10.1121/1.420324

\section{General rights}

Copyright and moral rights for the publications made accessible in the public portal are retained by the authors and/or other copyright owners and it is a condition of accessing publications that users recognise and abide by the legal requirements associated with these rights.

- Users may download and print one copy of any publication from the public portal for the purpose of private study or research.

- You may not further distribute the material or use it for any profit-making activity or commercial gain

- You may freely distribute the URL identifying the publication in the public portal 


\title{
Measurements and analysis of acoustic backscattering by elastic cubes and irregular polyhedra
}

\author{
Peter D. Thorne \\ Proudman Oceanographic Laboratory, Bidston Observatory Birkenhead, Merseyside LA3 7RA, \\ United Kingdom \\ Shaobo Sun \\ Department of Industrial Acoustics, Technical University of Denmark Building 425, DK-2800 Lyngby, \\ Denmark \\ Jingdong Zhang \\ School of Physics, University of Bath, Claverton Down, Bath BA2 7AY, United Kingdom \\ Irina Bjorno \\ Department of Industrial Acoustics, Technical University of Denmark, Building 425, DK-2800 Lyngby, \\ Denmark \\ Thierry Mazoyer \\ Metravid Research Development Service, 200 Chemin des Ormex, 69760 Limonest, France
}

(Received 22 August 1996; revised 30 May 1997; accepted 29 July 1997)

\begin{abstract}
Underwater acoustic studies of backscattering by submerged targets have generally focused on bodies with spherical and cylindrical symmetry. However, there are interests in scattering by objects which may be characterized by more angular features, with surfaces that tend to be composed of facets and edges. To investigate the scattering properties of such bodies, the backscattering by a number of elastic cubes, and irregularly shaped polyhedra, have been studied. Data were collected by measuring the band limited impulse response of the scatterers, using a broadband transducer, which operated as a transceiver, both transmitting and receiving signals. To present the scattering measurements nondimensionally a form function definition has been employed to normalize the backscattered signals. The normalized frequency has been expressed as $k a$, where $k$ is the acoustic wave number, and $a$ is a characteristic dimension of the scatterer. The cube observations covered a broad $k a$ range, $k a=3-34$, thereby encompassing the lower $k a$ scattering region where the cube can be considered to be nominally rigid, through to higher $k a$ values where it would be expected that the elastic properties of the cube become important. The measurements on irregular polyhedra are more limited in extent, however, the results are presented for comparison with the cube data. To compare the observations with predictions, computations of acoustic backscattering for both rigid and elastic cubes are presented. (C) 1997 Acoustical Society of America. [S0001-4966(97)04811-X]
\end{abstract}

PACS numbers: 43.30.Gv, 43.30.Ma, 43.30.Ft

\section{INTRODUCTION}

Measurements and theoretical works on the interaction of underwater sound with elastic targets of spherical and cylindrical geometries have established a detailed understanding of the scattering properties of such bodies. ${ }^{1-5}$ In particular the application of resonance scattering theory ${ }^{6-10}$ has clarified the component structure of the scattered signal in terms of background and resonant components. While the scattering by canonically shaped targets is therefore well understood, the scattering by objects which lack spherical or cylindrical symmetry is less developed, and there are open questions regarding the scattering characteristics and equivalent form function for nonspherical targets. To investigate some of these questions, the present paper examines the backscattering by cubes, and irregularly shaped polyhedra.

Advancing our understanding of the scattering of underwater sound by objects which are not canonical, but are more angular in form, with features composed principally of edges and facets, has been stimulated in part because of its possible application to the interpretation of the interaction of sound with marine suspensions. In recent years acoustic backscatter systems ${ }^{11-15}$ have been developed which can make detailed observations of near-bed suspended sediment processes. To extract suspended sediment parameters from the acoustic data entails an inversion procedure which is based on an assumed knowledge of the scattering characteristics of marine suspended sediments. To date the modeling of such particles has been based on spheres or fitting empirical algorithms to data sets. ${ }^{16-18}$ Such pragmatic approaches have been very valuable, and they form the cornerstone of the acoustic inversion. However, as seen in Fig. 1, scanning electron micrographs show sediments composed of sand to contain a substantial proportion of particles with surfaces which have a tendency to be angular, and have a number of facets and edges. As a step toward identifying the scattering properties of such bodies, the backscattering characteristics of elastic cubes have been investigated in detail in the present work. Further, some limited measurements on irregular polyhedra are presented. 


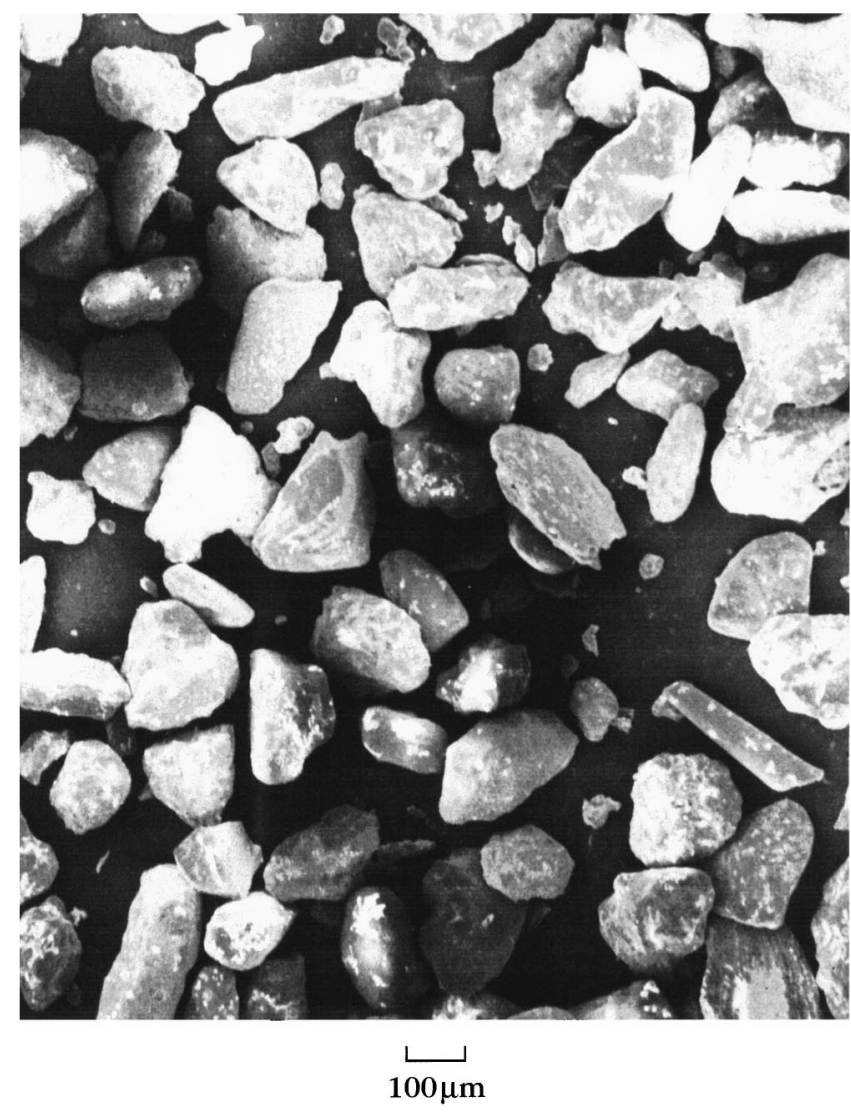

FIG. 1. Scanning electron micrography of quartz sand sediment particles.

To obtain the backscatter measurements a calibrated broadband transceiver was used, both transmitting the incidence signal toward the target, and receiving the backscattered signal from the target. The transducer transmitted a short wide-band pulse, and the band limited impulse response of the target was recorded. Data were collected on four different size cubes which covered a $k a$ region of 3-34, and on three irregular polyhedra between $k a=7$ and 25 .

Modeling the scattering characteristics of cubes and polyhedra is a relatively complex task because the wave equation is not separable for these geometries. Therefore analytical solutions are not available, and numerical methods are the only techniques presently able to predict the scattered field by such targets. In the present study theoretical approaches are employed which use the boundary element method (BEM) for rigid scatterers, and a combined BEM and finite element method (FEM) for elastic scatterers. Even with increasing access to powerful computing facilities, the computations are protracted, and the solutions tend to become more problematic at higher $k a$ values. Therefore the calculations in the present work have been limited to upper values of $k a=20$ and $k a=16$, for the rigid and elastic cases, respectively.

\section{NUMERICAL SCATTERING MODELS}

In order to tackle the problem of scatterers in which the wave equation is not separable, numerical techniques remain the principal method for obtaining the scattered field. In the present study both the rigid and elastic response of a cube have been computed. To calculate the rigid response, a boundary element method has been applied to a rigid immovable cubic mesh in the frequency domain. One primary objective was to cover a broad ka range, and therefore particular care was taken to overcome the nonunicity of the standard BEM solution at certain characteristic frequencies. The solution employed was to use a Helmholtz gradient formulation (HGF), which consists of combining the Helmholtz integral equation with its normal derivative form. ${ }^{19,20}$ Further, the utilization of variational formalism, ${ }^{21}$ instead of the conventional collocation technique, leads to significant numerical simplification when computing the highly singular integrals involved in BEM. To compute the elastic response of a cube advantage was taken of a recently developed software package PAFEC. ${ }^{22}$ PAFEC models the scattering problem using a combined FEM and BEM model. Finite elements are used to characterize the vibration of the elastic body, and boundary elements describe the radiation or scattering from the body. Even with the package, care was required in the formulation of the cube scattering problem, and an examination was needed to take advantage of available symmetries for particular cube scattering configurations, to optimize the intensive computations needed for the elastic case.

The cubes and polyhedra investigated were composed of iron. At the time of the experiments the material had been thought to be stainless steel, but later metallurgic analysis showed this not to be the case. This has introduced a degree of complication into the comparison between the observations and the computed elastic case because the elastic properties of iron tend to be less consistent than that of stainless steel. However, this said, useful comparisons have been made both with the experimental data and with the calculations for the rigid cube case. The parameters used for the elastic calculations were obtained from tabulated values for iron which gave compressional and shear wave velocities, respectively, of $5957 \mathrm{~m} \mathrm{~s}^{-1}$ and $3224 \mathrm{~m} \mathrm{~s}^{-1}$, and a density of $7860 \mathrm{~kg} \mathrm{~m}^{-3}$. The density and sound velocity of water used were respectively $1000 \mathrm{~kg} \mathrm{~m}^{-3}$ and $1460 \mathrm{~m} \mathrm{~s}^{-1}$.

\section{EXPERIMENTAL ARRANGEMENT}

Measurements of the form function ${ }^{23}$ were conducted in a small laboratory test tank with dimensions of $1.5 \times 0.5$ $\times 0.5 \mathrm{~m}$. The acoustic axis was directed along the center of the longest dimension of the test tank. A diagram of the instrumentation is shown in Fig. 2. The transducer used acted as a transceiver both transmitting and receiving signals. The transducer operated at a center frequency of $546 \mathrm{kHz}$ and had an operating bandwidth between 300 and $800 \mathrm{kHz}$. Measurements were conducted by transmitting a single cycle tone burst at the center frequency, and the backscattered signal was gated, amplified, filtered, coherently averaged, digitized, and recorded. The voltage applied to the transducer was also digitized and recorded. The transmitting and receiving sensitivities of the transducer were measured using a reference hydrophone, and further self-reciprocity calibrations, and measurements ${ }^{24}$ of the specular echo from a sphere confirmed the sensitivities. The transfer function of the electronics was also measured. 


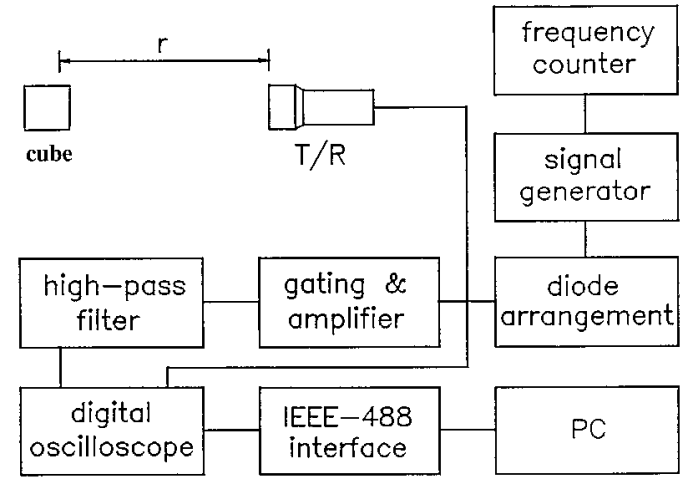

FIG. 2. Instrumentation used for the scattering measurements.

The experimental form function, $f$, for a threedimensional scatterer can be defined as

$$
f=\frac{2 r}{a_{c f}} \frac{P_{s}(\nu)}{P_{i}(\nu)}
$$

where $r$ is the distance between the center of the scatterer and the transducer, $P_{s}(\nu)$ is the spectrum of the scattered signal, $P_{i}(\nu)$ is the spectrum of the incident signal, $\nu$ is frequency, and $a_{c f}$ is a characteristic dimension. In a recent study ${ }^{25}$ it has been shown that the average projected area for any convex scatterer is a quarter of its surface area, and this leads to the correct value for $a_{c f}$ of $a_{c f}=l(3 / 2 \pi)^{1 / 2}$, where $l$ is the side length of a cube. The appropriate value to normalize the frequency axis is less clear, however, comparisons of the form function for a rigid sphere, and a rigid cube ${ }^{25}$ indicate that using the radius of a sphere having the same volume as the cube has advantages. This value is therefore given by $a_{c k}=l(3 / 4 \pi)^{1 / 3}$, and the nondimensional frequency axis is given by $k a_{c k}$. by

Re-expressing in terms of the measured signal $f$ is given

$$
f=\frac{2 r^{2}}{a_{c f}} \frac{V_{s}(\nu)}{V_{i}(\nu) G(\nu) S_{t r}(\nu)}
$$

where $V_{s}(\nu)$ is the spectrum of the backscattered voltage, $V_{i}(\nu)$ is the spectrum of the voltage applied to the transceiver, $G(\nu)$ is the gain of the electronics system, and $S_{t r}(\nu)$ is the transceiver sensitivity. To assess the validity of the form function measurements collected with the present system, observations of the scattering characteristic of a 12.5$\mathrm{mm}$-radius stainless steel sphere were recorded, and the form function evaluated using Eq. (2). Comparison with theoretical predictions are shown in Fig. 3. The similarity between the measured and predicted form function confirms the accuracy of the system calibration.

\section{MEASUREMENTS AND ANALYSIS}

Backscatter data were collected for varying angles of incidence and different cube configurations. Figure 4 shows the main configurations used, with the cube face-on, edgeon, and at an arbitrary configuration to the incoming signal. The cubes were rotated about the axis as shown and the variation in the backscattered form function with angle measured. The majority of data shown in the present work are associated with the face-on configuration [Fig. 4(a)], at varying angles of incidence, although to estimate the mean backscatter form function for a cube, measurements at different angles were also collected using the configurations shown in Fig. 4(b) and (c). The irregular shaped polyhedra data were collected by taking measurements at a number of angles of incidence (simply referenced to a starting position and rotated), and configurations.

Measurements of the backscattered signal from a cube in the time domain are shown in Fig. 5. To illustrate the form and duration of the signal employed Fig. 5(a) shows the specular component of the backscattered signal from a 12.5mm-radius sphere. Figure 5(b)-(d) shows the backscattered signal for the face-on configuration at $\theta=0^{\circ}$ (normal incidence), $10^{\circ}$, and $45^{\circ}$, for a cube with 8 -mm-length sides. The $0^{\circ}$ shows a time series echo that is comparable to the specular echo from the sphere, with the signal being primarily composed of a simple single pulse. By $10^{\circ}$ the amplitude of the backscattered signal has reduced by about a factor of 5 ,

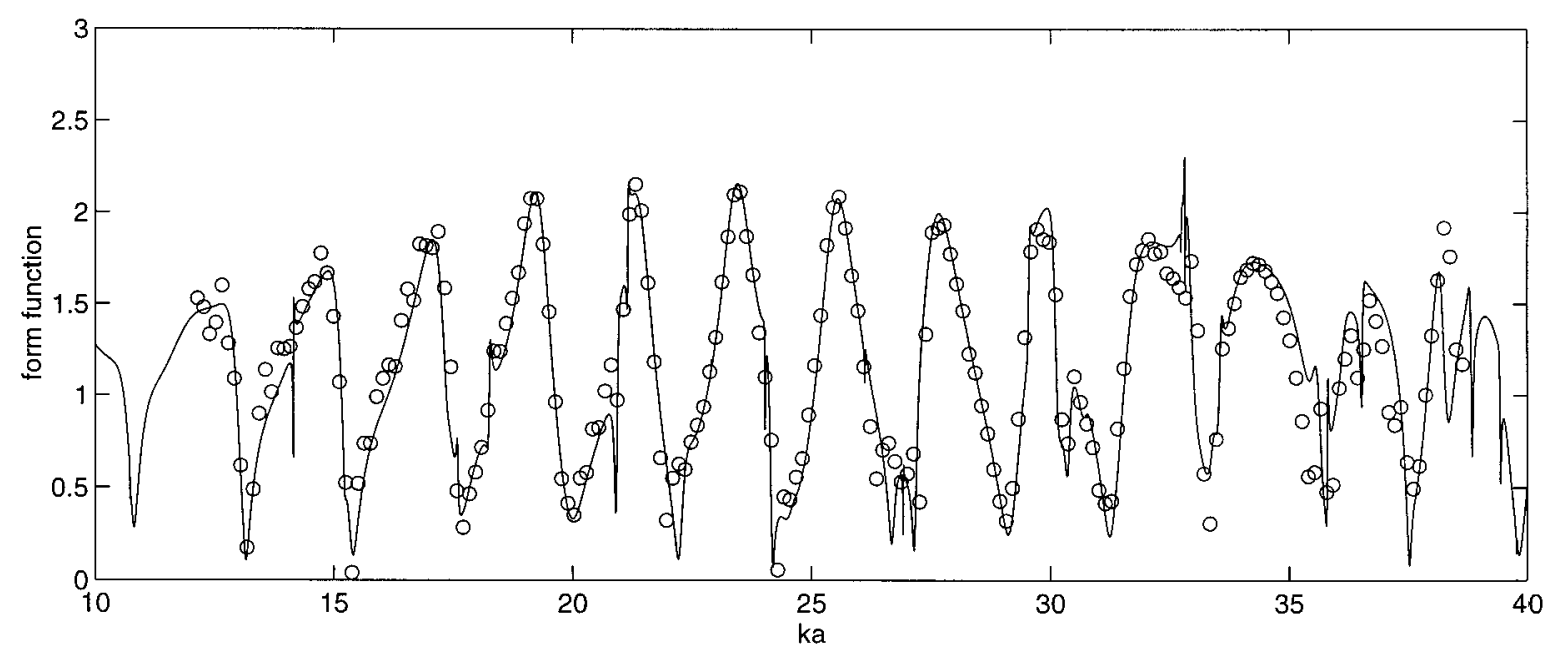

FIG. 3. Comparison of the measured $(\bigcirc)$ and the predicted $(-)$ form function for a 12.5 -mm-radius stainless steel sphere. 
(a)

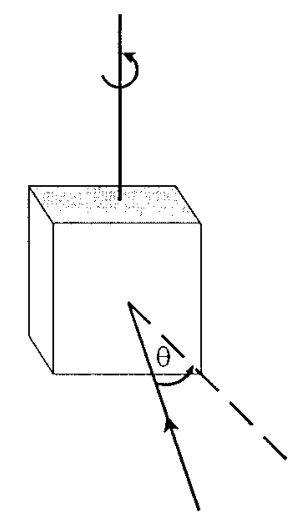

(b)

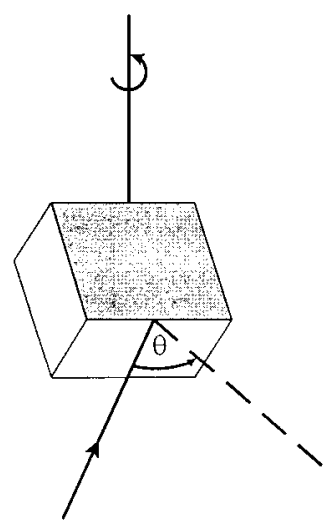

(c)

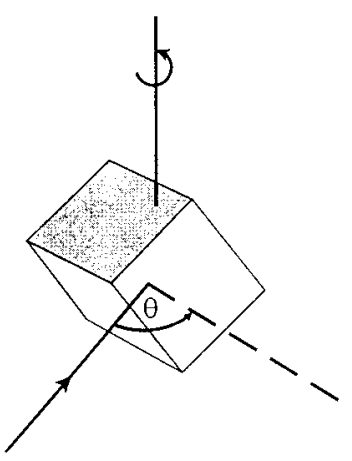

FIG. 4. Various configurations of the cube mounting. (a) Face-on; (b) edgeon; and (c) arbitrary configuration.

and there is a structured echo with a duration which is significantly greater than that for $0^{\circ}$. By $45^{\circ}$ (which is the same as the normal incidence edge-on case) the signal amplitude is now around one-tenth of the $\theta=0^{\circ}$ case. The signal has an initial arrival which is considered to be due to the leading edge, and this is followed by the bulk of the signal. The latter is relatively complex in structure and has a duration which is somewhat longer than the $10^{\circ}$ result.

From the time domain waveforms the backscattered form functions have been computed using Eq. (2). The normal incidence results, $\theta=0$, for the face-on configuration are shown in Fig. 6. The data were collected on cubes with side lengths of $4(+), 8(\bigcirc), 12\left(^{*}\right)$, and $16(\times) \mathrm{mm}$. Unlike sphere scattering, which results in a form function which oscillates about unity with increasing $k a$, owing to its flat surface, the form function for each cube is observed to steadily increase with $k a$. Superimposed upon this general
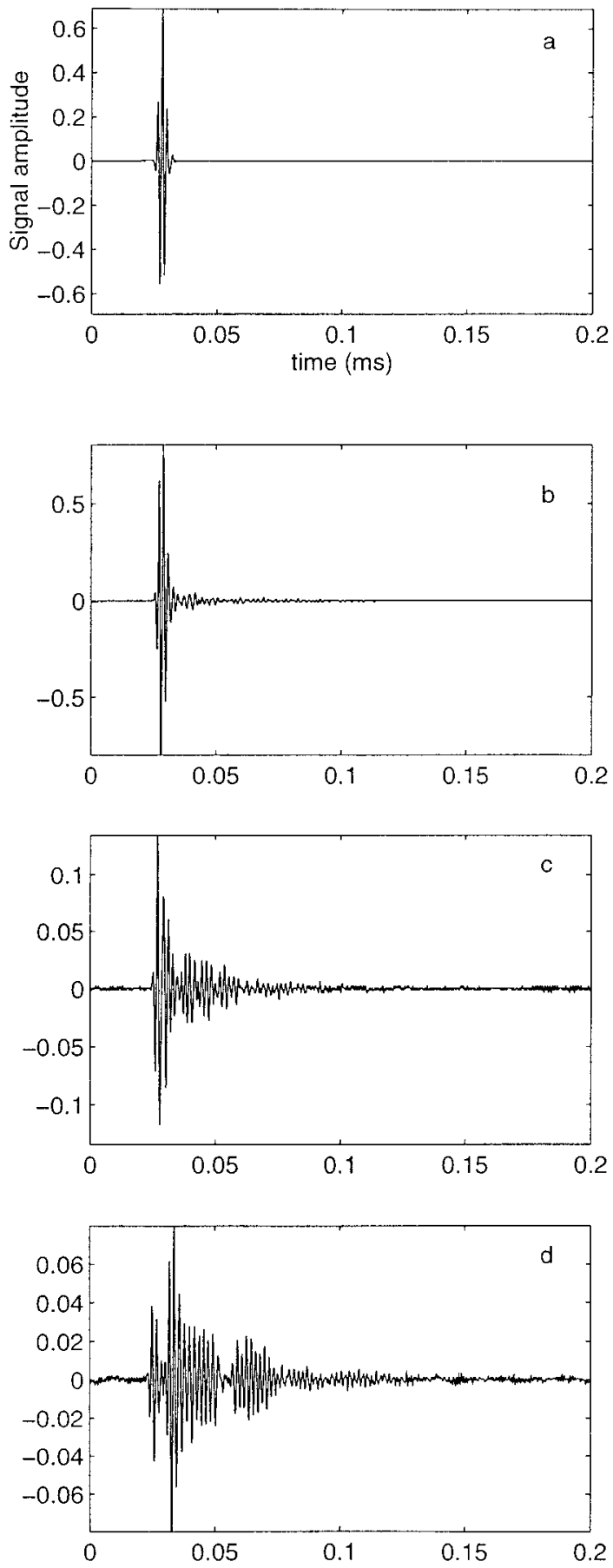

FIG. 5. Backscattered time series measurements. (a) Specular echo from a 12.5-mm-radius sphere. Backscattered echo for the face-on configuration for a cube at: (b) $\theta=0^{\circ}$; (c) $\theta=10^{\circ}$; and (d) $\theta=45^{\circ}$.

trend are a series of dips, and the $k a$ location of these dips are consistent for the different cubes. Two theoretical form functions are compared with the data in Fig. 6(a), the rigid immobile cube (-.-), and the elastic cube (-). It can be seen that the rigid case compares well with the data in the low $k a_{c k}$ region. This is as might be expected for an iron cube, since a rigid model should be a reasonable approximation at low $k a_{c k}$ values. Above a $k a_{c k} \approx 6$ the rigid predictions are in agreement with the general trend of the data although the dips in the form function are not replicated. The elastic re- 

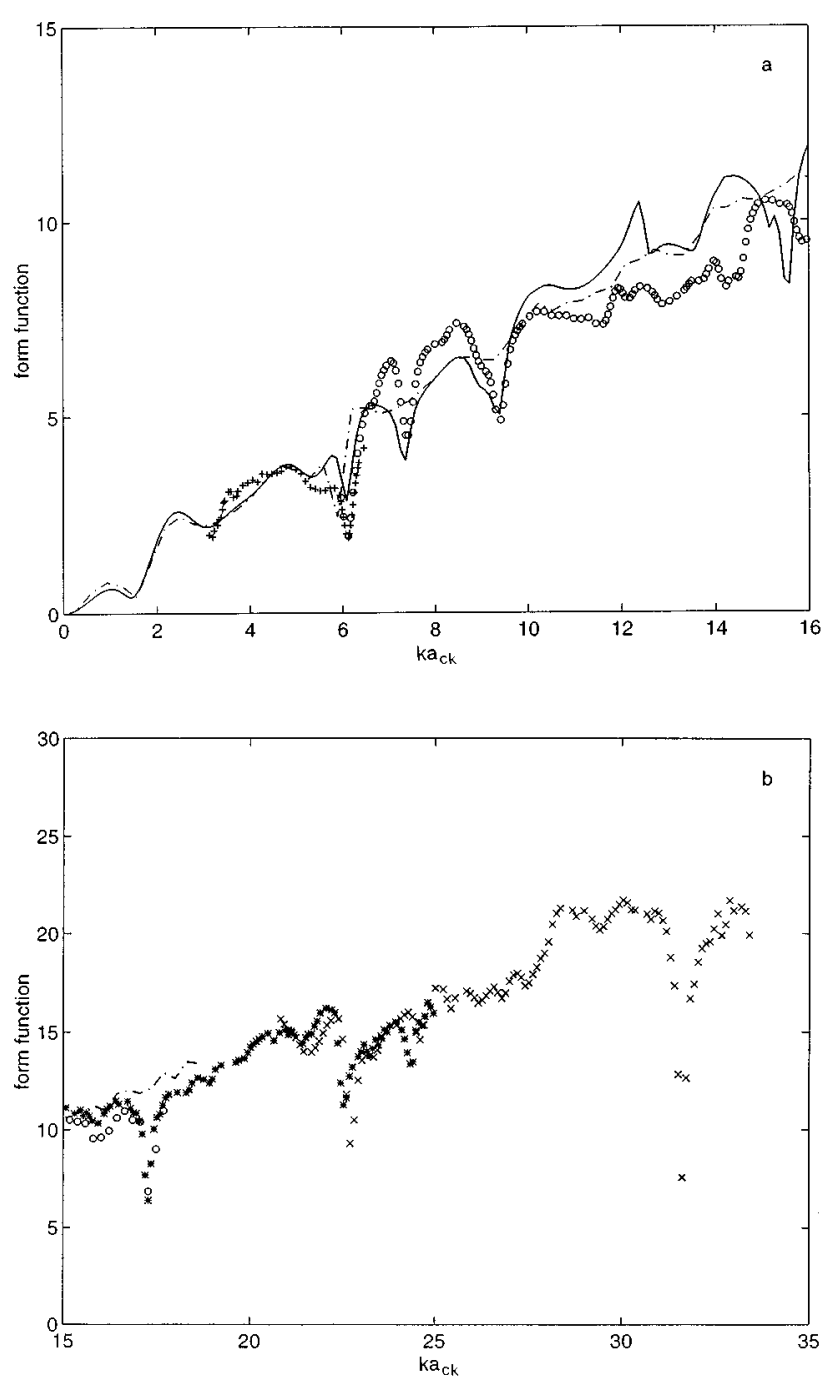

FIG. 6. Comparison of the measured and the predicted form function at $\theta$ $=0^{\circ}$ for the face-on configuration. Measurements were taken using $4(+), 8$ $(\bigcirc), 12(*)$, and $16(\times) \mathrm{mm}$ sized cubes. Predictions were computed using rigid (-.-) and elastic (-) models for a cube.

sults, however, show both general agreement with the data, and the dips in the form function at $k a_{c k}=6,7.4$, and 9.4 are replicated. There does appear to be some discrepancy between predictions and observations just below $k a_{c k}=6$, and for $k a_{c k}>10$. This could be associated with the uncertainty in the estimated values for the compressional and shear wave velocity of the iron used to form the cubes. However, because of the extensive computations required to obtain the elastic results, up to periods of several days, it is presently impractical to run a series of scenarios with varying sound speeds to investigate the details of the difference. Also the precision of the alignment, considered to be of the order of $1^{\circ}$ in both the rotational and vertical direction, may be influencing the level of the higher $k a$ values. Notwithstanding these limited differences between theory and experiment, the main features of the normal incidence data are well represented using the elastic formulation for cube scattering. In Fig. 6(b) results are presented up to $k_{c k}=33$. These continue to show an increase in form function with $k_{c k}$ with a number of dips. There are no elastic calculations for this regime,

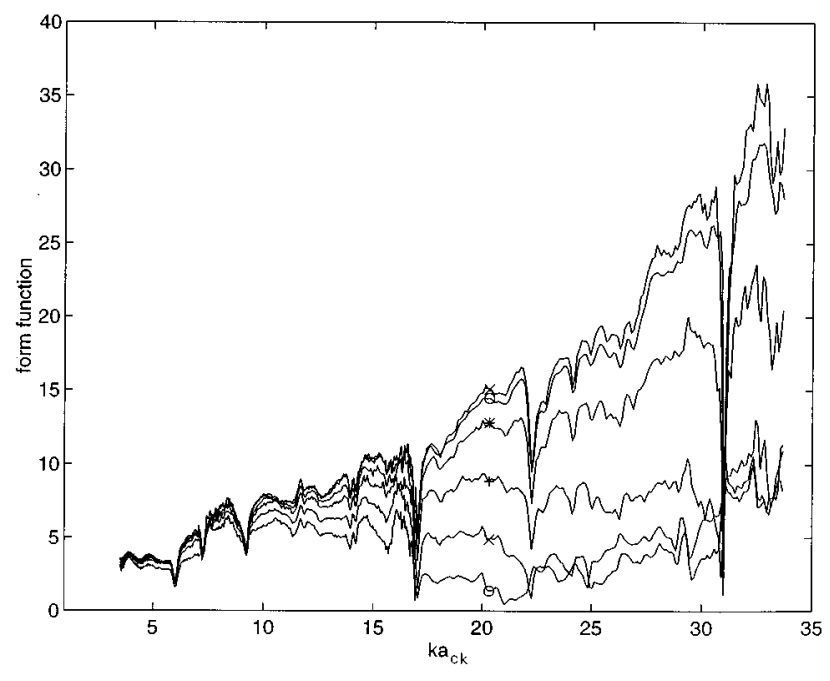

FIG. 7. Variation in the form function at near-normal incidence angles for the face-on configuration. $x$ (upper curve) $-\theta=0^{\circ}, \bigcirc-\theta=1^{\circ}, *-\theta=2^{\circ}$, $+-\theta=3^{\circ}, \times-\theta=4^{\circ}$, and $\bigcirc$ (lower curve $)-\theta=5^{\circ}$.

although values for the rigid case are presented up to $k_{c k}=20$. Again the rigid case provides the general form but not the notable dips observed in the data. Since from Fig. 6(a) these dips are replicated in the elastic case, it would appear that dips are associated with modes of vibration of the cube which interfere destructively with the sound reflected from the surface of the cube, the latter being represented by the rigid calculation. To investigate the angular stability of the form function dips observed in the theory and the experiment at $\theta=0$, measurements were obtained off normal incidence. Figure 7 shows the outcome from some of these observations for $\theta=0^{\circ}-5^{\circ}$. It can be clearly seen that although the amplitude of the form function reduces rapidly off normal incidence, there is coherence in the location of the dips out to about $5^{\circ}$.

Figures 8-11, respectively, show measurements of the backscattered form function at $5^{\circ}, 10^{\circ}, 30^{\circ}$, and $45^{\circ}$ for the

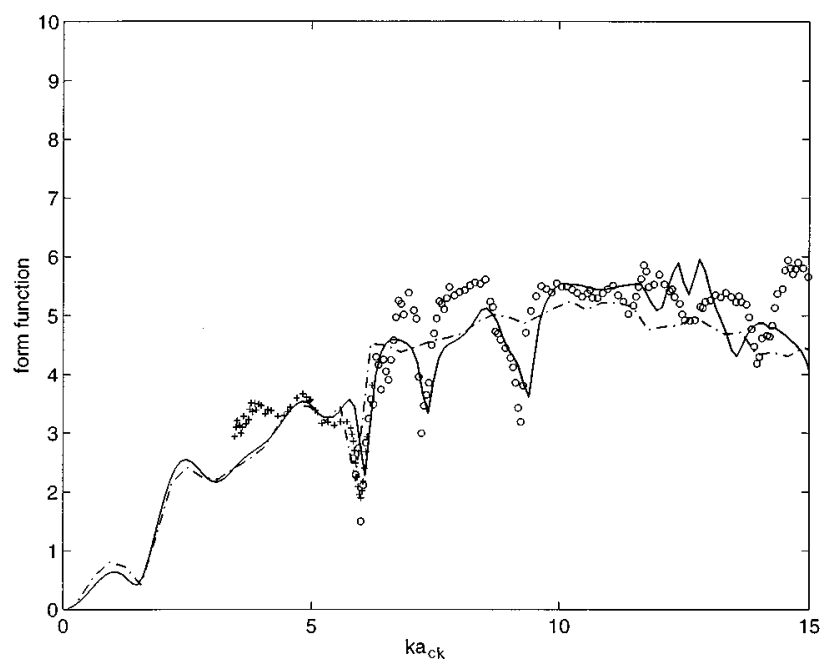

FIG. 8. Comparison for the face-on configuration at $\theta=5^{\circ}$ of the form function computed for rigid (-.-) and elastic (- cubes, and measured using $4(+)$ and $8 \mathrm{~mm}(\bigcirc)$ sided cubes. 


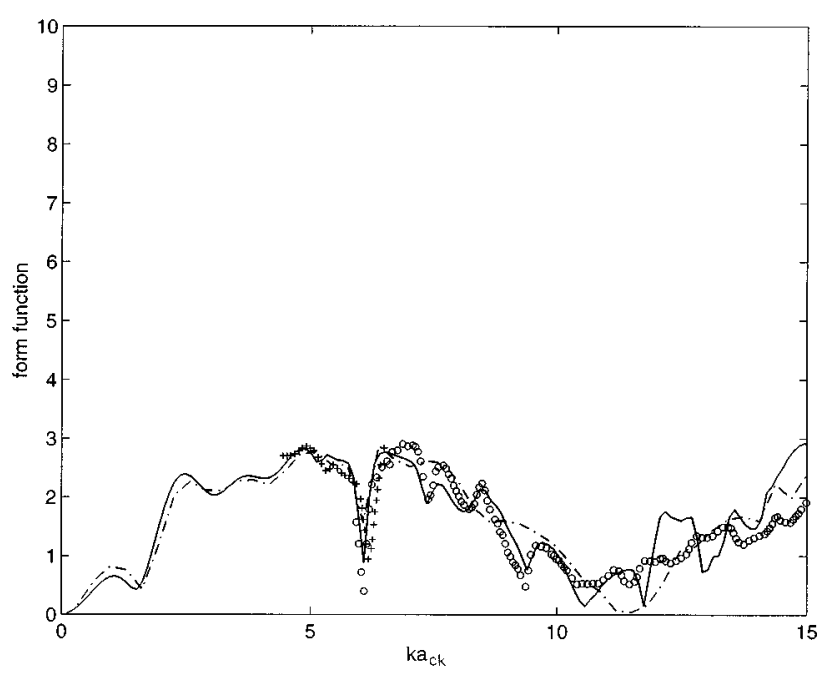

FIG. 9. Comparison for the face-on configuration at $\theta=10^{\circ}$ of the form function computed for rigid (-.-) and elastic (- $(-)$ cubes, and measured values using $4(+)$ and $8 \mathrm{~mm}(\bigcirc)$ sided cubes.

face-on configuration. The experimental data shown in Fig. 8 have form function values nominally between four and seven, with a series of identifiable dips, which as noted above, are present for all near-normal angles of incidence. For the $5^{\circ}$ case the rigid computations provide a reasonable estimate for the general shape of the form function, however, the detailed variability associated with the cube being elastic is not reproduced. The elastic case provides results which are comparable with the observation, and with the dips in the form function being present. The results shown in Fig. 9 for a $10^{\circ}$ angle of incidence show a different structure to those at $5^{\circ}$, with a reduction in the general level of the form function near $k a=10-12$. Predictions based on rigid and elastic cubes are compared with the data. The rigid results again provide the general form of the data, while it requires the elastic solution to replicate the detailed variability in the observations, although beyond $k a_{c k}=10$ there are differences between prediction and observation, these are probably due

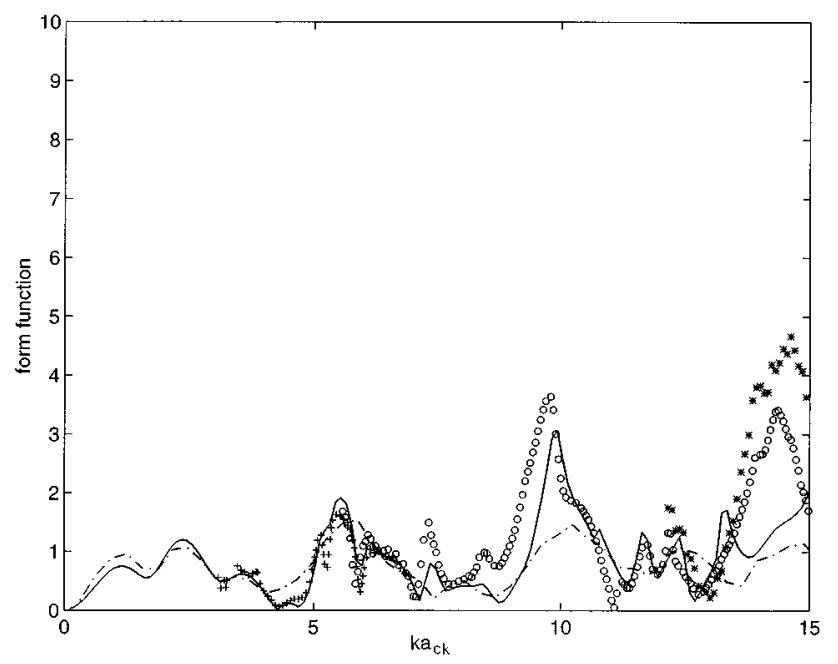

FIG. 10. Comparison for the face-on configuration at $\theta=30^{\circ}$ of the form function computed for rigid (-.-) and elastic (- cubes, and measured using $4(+), 8(\bigcirc)$, and $12 \mathrm{~mm}(*)$ sided cubes.

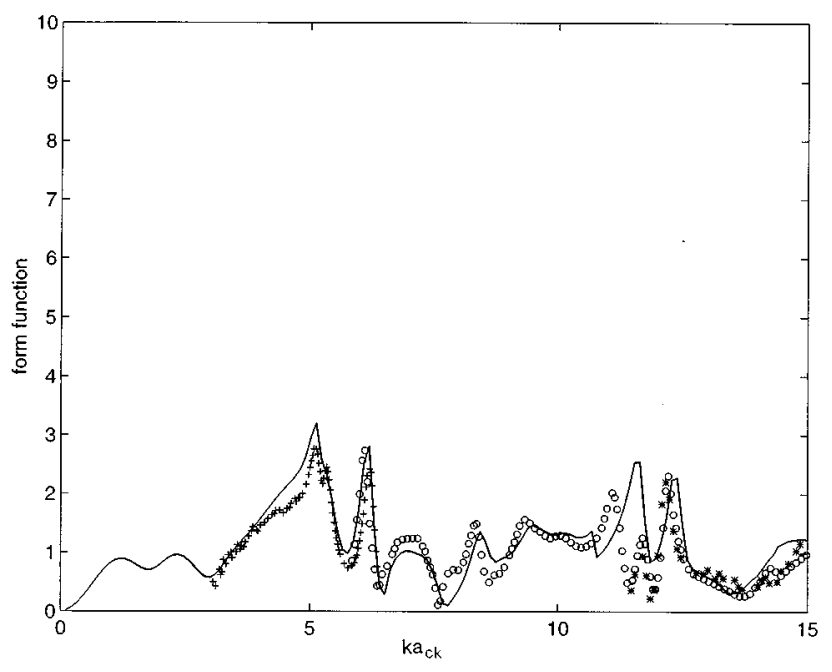

FIG. 11. Comparison for the face-on configuration at $\theta=45^{\circ}$ (equivalent to the edge-on configuration at $\theta=0^{\circ}$ ) of the form function computed for an elastic cube (—), and measured values using $4 \mathrm{~mm}(+), 8 \mathrm{~mm}(\bigcirc)$, and $12(*)$ mm sided cubes.

to uncertainties in the compressional and shear wave velocities for iron. The $30^{\circ}$ results are shown in Fig. 10. The structure of the form function is quite variable with a series of peaks and troughs in the data. The general background trend with a number of dips superimposed upon it observed at the near-normal angles is not seen, and the form function is generally more complex. To compare the observations with the predictions, computation for a rigid and an elastic cube were carried out. There is seen to be reasonable agreement between the rigid calculations and measurements for $k a \leqslant 7$, however, at higher $\mathrm{ka}$ values the comparison between the rigid computations and the data is poor due to the onset of the elastic characteristics of the cube. The elastic computations show significantly improved agreement with the data, with, for example, the peak in the form function near $k a_{c k}$ $=10$ being replicated, albeit at a slightly higher $k a_{c k}$ value. Figure 11 shows measurements and computed predictions for the elastic case at $\theta=45^{\circ}$; this configuration is equivalent to the configuration in Fig. 4(b) at $\theta=0^{\circ}$. The data show notable peaks in the form function around $k a_{c k} \approx 5$ and $k a_{c k}$ $\approx 6$, followed by a form function structure which is variable in form. The elastic calculations are seen to be in reasonable agreement with the data for $k a_{c k}=0-15$ for this angle. The fact that for some angles the comparison between the elastic and measured data are better than at other angles possibly indicates a degree of anisotropy in the material, this would not necessarily be unexpected for iron. At present for the elastic case computational periods are too extensive to iterate the compressional and shear wave velocities, and optimize the comparison at each angle.

The form function comparisons at particular angles allow the theory and experimental results to be examined in detail, however, they do not provide a global view of the scattering characteristics for a cube. To present a more general impression of the scattering properties, 3-D plots of the form function for 4-, 8-, and 16-mm-sided cubes are shown in Fig. 12(a)-(c). These were obtained using the face-on configuration, and the cube rotated from $\theta=0^{\circ}$ to $45^{\circ}$ in $1^{\circ}$ 


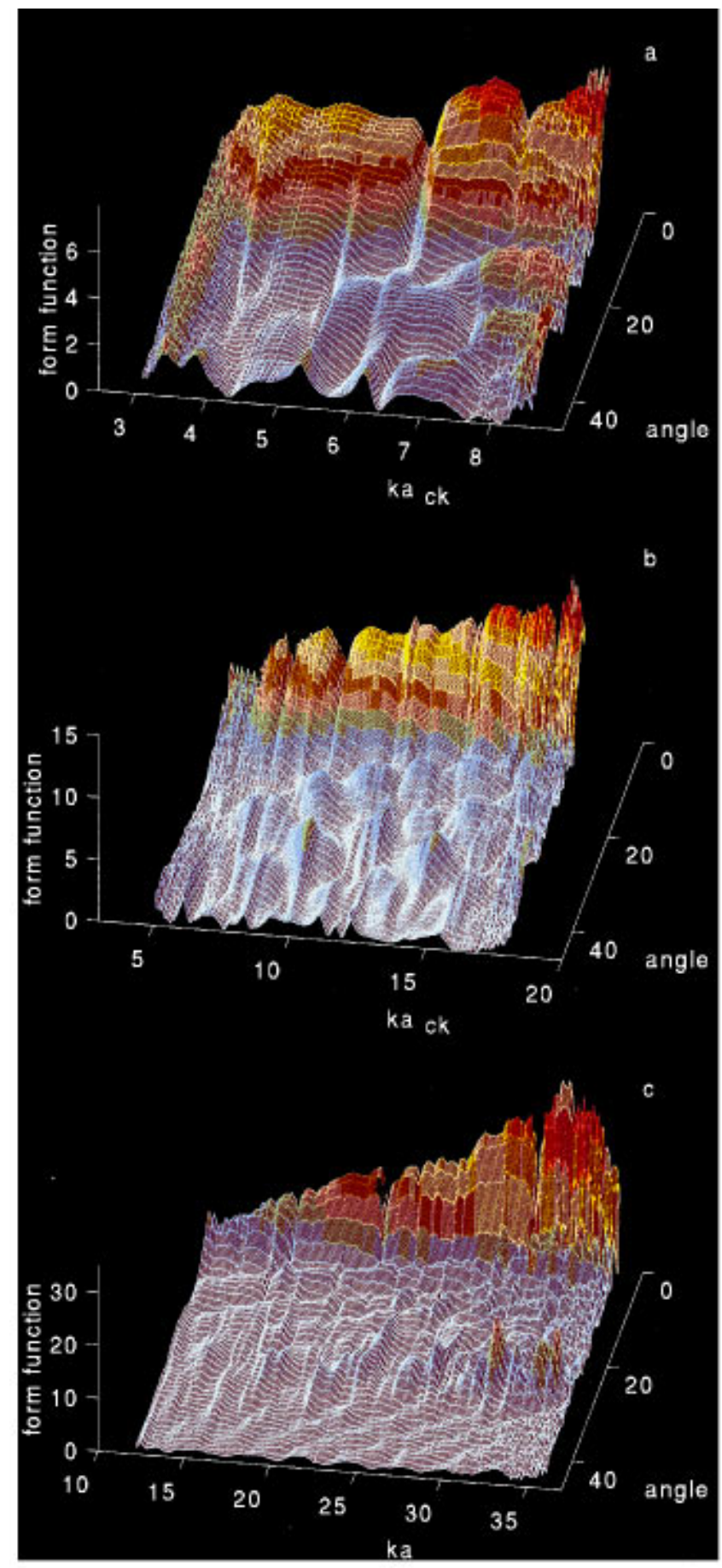

FIG. 12. Measurements of the variation of the form function for the face-on configuration with $k a_{c k}$ and $\theta$ for (a) 4, (b) 8, and (c) $16 \mathrm{~mm}$ sided cubes.

steps. Near normal incidence each plot clearly shows high form function values with readily identifiable dips. These high values rapidly reduce away from normal incidence, and beyond about $10^{\circ}$ the form function has an undulating structure both in $\theta$ and $k a_{c k}$, with values which typically oscillate between approximately 0 and 2 . The physical interpretation of such figures is difficult at present because of the lack of an analytical representation of the cube scattering, and the long periods required to compute the results. However, the plots do provide a broad picture of cube scattering for the face-on configuration.
In part, the stimulation for developing a description of the scattering properties of a cube was an interest in the scattering properties of suspensions of marine sediments. It was therefore considered useful to examine the mean scattering properties of a cube. This was carried by taking a number of measurements using the three configurations shown in Fig. 4. A total of 107 form function measurements were taken on an 8-mm-side cube, and the average result from this data set is shown in Fig. 13. This result shows an average form function for the data set which is nominally centered about unity, with a number of notable peaks between $k a_{c k}$ $=6$ and 10, and a steady oscillation in the form function for $k a_{c k}>10$. The peaks in the $k a_{c k}=6-10$ region do appear to be genuine features; they were noticeable in the individual form functions for some of the arbitrary configurations. The regular oscillations above $k a_{c k}=10$ are close in amplitude to the normalized error in the mean, which for 107 measurements was 9.7\%; however, the regularity does suggest it may well be an actual feature. Two curves are compared with the data, i.e., the form function for a rigid sphere $(\cdots)$, and the average form function for a rigid cube (-.-). It is interesting to note that the theoretical form functions are comparable, and in particular the feature of a diffraction type oscillation for the average form function for a cube below $k a_{c k}=5$ is readily noticeable. Recent measurements ${ }^{25}$ on a cube in this low $k a_{c k}$ regime have clearly identified this structure. The rigid cube calculations, which were computed for 36 configurations, give approximately the same level as the observations, although the peaks are not observed and the higher $k a_{c k}$ oscillations are not readily decernable. The former is ascribed to the model being rigid, while the latter may be due to the relatively coarse value for the $k a_{c k}$ interval which was 0.31 .

The irregular polyhedra were formed by taking iron cubes, and filing the edges until the shape was comparable to the sand particles shown in Fig. 1. Both the surface area and volume of the irregular polyhedra were measured to appropriately normalize the data. Measurements were taken on three irregular polyhedra having $a_{c k}$ values of 4, 6.3, and 8.1 $\mathrm{mm}$. Data were obtained for three configurations, and eight different angles of incidence for each polyhedron, and the mean form function for each polyhedron calculated. The average form functions was obtained by taking the average of the three mean form functions. The final result is presented in Fig. 14 and this shows the irregular polyhedra to have an average form function which is nominally uniform, and with a magnitude close to unity. Two curves are compared with the data, those of a rigid sphere $(\cdots)$ and a rigid icosahedron (-.-). The icosahedron was chosen because the irregular polyhedra had of the order of 20 sides, and its symmetry was amenable to computation. The computational results for the icosahedron are seen to be very similar to those of a rigid sphere with the diffraction oscillation clearly delineated. The rigid sphere could be thought of as a rigid polyhedron with an infinite number of facets; therefore as the number of facets on a polyhedron increase it might be expected that the solution would tend toward that of a sphere. Comparison of the computations with the observations show good agree- 


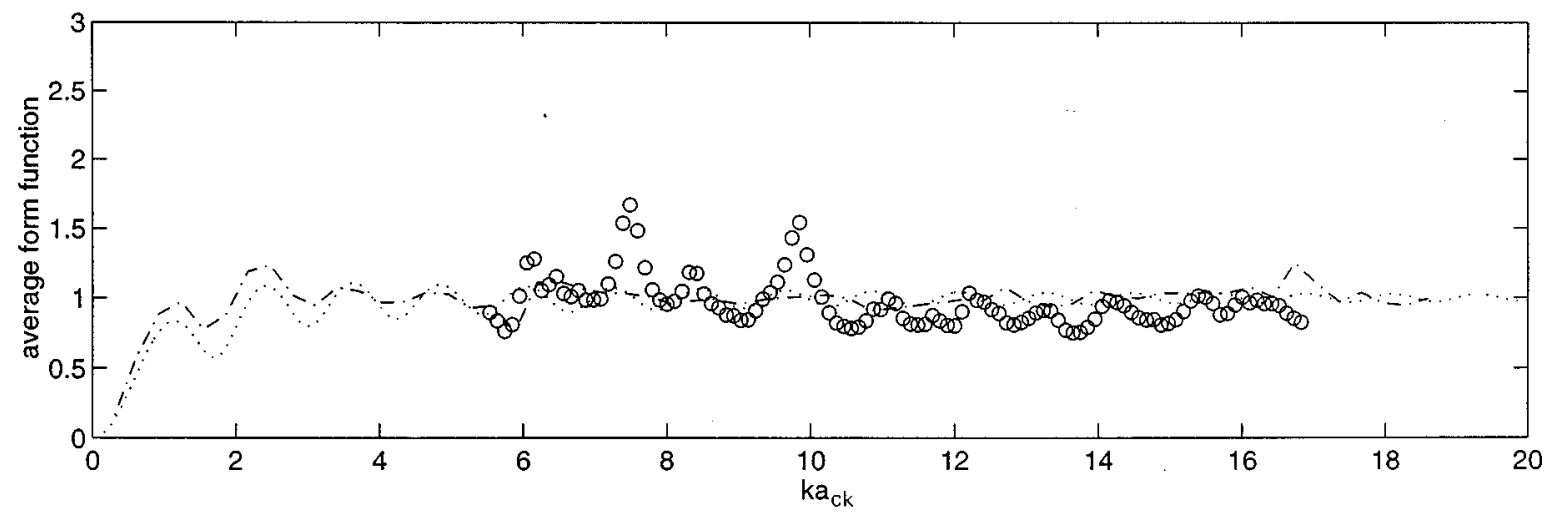

FIG. 13. Comparison of the average form function computed for a rigid cube (-.-) and a rigid sphere ( $\cdots)$, with measured values for a $8 \mathrm{~mm}(\bigcirc)$ sided cube.

ment between the predicted and the measured average form functions.

\section{CONCLUSIONS}

A series of measurements on the backscattering by submerged elastic cubes have been conducted, and the results formulated nondimensionally using the form function definition, with a characteristic dimension defined by the projected surface area. The observations show a steadily increasing form function with $k a_{c k}$ for normal incidence with the face-on configuration. Rotation off normal incidence results in a rapid reduction in the amplitude of the form function, although there is a degree of coherence in the structure of the form function up to about $5^{\circ}$. Beyond about $5^{\circ}$ the composition of the form function becomes more complex, especially at the higher $k a_{c k}$ values when the elastic nature of the cube becomes important. To provide a global sense of these relatively complex scattering characteristics, 3-D plots have been generated, and these provide some impression of the overall $k a_{c k}-\theta$ structure.

To theoretically describe the scattering properties of a cube, numerical computations using both rigid and elastic models have been evaluated. The rigid predictions provide solutions which are valid at low $k a_{c k}$, and give a first approximation to the general structure of the form function over a broad $k a_{c k}$ range at near-normal incidence for the face-on configuration. To describe the detailed variability in the form function, and off normal incidence angles requires the elastic model to be run, and this does indeed provide significantly improved agreement between the predictions and the observations. Even with the elastic model there are discrepancies between computed values and measured data, and these are considered to be associated with uncertainties in the appropriate values for the compressional and shear wave velocities for iron.

Finally configuration and angular averaged form functions have been presented for a cube and irregular polyhedra. These show form functions which nominally have amplitudes of the order of unity, although in the cube case a persistent regular structure is observed. A number of predictions have been made for the average form function response, with rigid sphere, cube, and icosahedron shapes being computed. An interesting feature of the computations is the diffraction type oscillations observed in the cube and icosahedron form functions in the low $k a_{c k}$ region which appear comparable in periodicity to those on a sphere generated by creeping waves. Such oscillations have been recently observed experimentally $^{25}$ for a cube in the $k a_{c k}=0-5$ region, and thereby validate the predictions. It therefore appears that there could be some kind of specular, and creeping wave interaction which is emerging through the mean form function that is comparable with that of a sphere. Comparison of

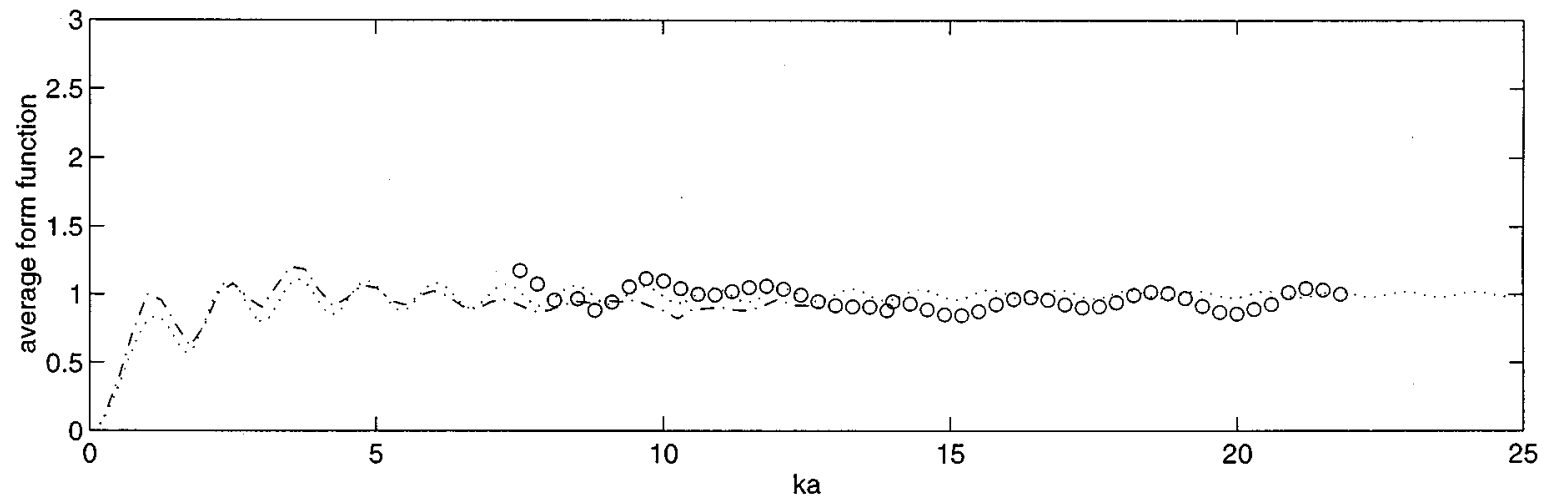

FIG. 14. Comparison of the average form function computed for a rigid icosahedron (---) and a rigid sphere ( $\cdots)$, with measured values $(\bigcirc)$ obtained using irregular polyhedra with $a_{c k}$ values of $4,6.3$, and $8.1 \mathrm{~mm}$. 
the computed form functions with the data show reasonably consistent results, and the results indicate why the sphere based scattering models for sediment scattering yield reasonable results. To first order the mean form function for rigid polyhedra, (which from Fig. 1 would appear to be a more appropriate description for sandy sediments) are similar to that of a rigid sphere.

\section{ACKNOWLEDGMENTS}

This work was supported by the European community under its MAST programs, and by the coastal protection division of MAFF, UK. P.D.T. became involved in this work during a visit to the Department of Industrial Acoustics at the Technical University of Denmark, and he would like to thank Professor Leif Bjorno for his invitation and support. Thanks also go to Dr. Victor Humphrey and Dr. Paul Chinnery, of Bath University, U.K., for a number of interesting discussions on scattering problems.

${ }^{1}$ J. J. Faran, "Sound scattering by solid cylinders and spheres," J. Acoust. Soc. Am. 23, 405-418 (1951).

${ }^{2}$ W. G. Neubauer, R. H. Vogt, and L. R. Dragonette, "Acoustic reflections from elastic spheres. I. Steady-state signals," J. Acoust. Soc. Am. 55, 1123-1129 (1974).

${ }^{3}$ P. L. Marston, "GTD for backscattering from elastic spheres and cylinders in water and the coupling of surface elastic waves with the acoustic field," J. Acoust. Soc. Am. 83, 25-37 (1988).

${ }^{4}$ R. Hickling, R. K. Burrows, and J. F. Ball, "Rotational waves in the elastic response of spherical and cylindrical acoustic targets in water," J. Acoust. Soc. Am. 89, 971-979 (1991).

${ }^{5}$ R. H. Hackman, G. S. Sammelmann, K. L. Williams, and D. H. Tivett, "A reanalysis of the acoustic scattering from elastic spheroids," J. Acoust. Soc. Am. 83, 1255-1266 (1988).

${ }^{6}$ L. Flax, L. R. Dragonette, and H. Uberall, "Theory of elastic resonance excitation by sound scattering," J. Acoust. Soc. Am. 63, 723-731 (1978).

${ }^{7}$ G. C. Gaunaurd and H. Uberall, "RST analysis of monostatic and bistatic acoustic echoes from an elastic sphere," J. Acoust. Soc. Am. 73, 1-12 (1983).

${ }^{8}$ W. G. Neubauer, "Acoustic reflection from surfaces and shapes," published by Naval Research Laboratory, Washington, DC (1986).

${ }^{9}$ M. F. Werby and G. C. Gaunard, "Resonance scattering from submerged elastic spheroids of high aspect ratios and its three-dimensional interpretation," J. Acoust. Soc. Am. 88, 951-960 (1990).

${ }^{10}$ D. Decultot, F. Lecroq, G. Maze, and J. Ripoche, "Acoustic scattering from a cylindrical shell bounded by hemispherical end caps. Resonance interpretation with surface waves propagating in cylindrical and spherical shells," J. Acoust. Soc. Am. 94, 2916-2923 (1993).

${ }^{11}$ A. E. Hay and J. Sheng, "Vertical profiles of suspended sand concentration and size from multifrequency acoustic backscatter," J. Geophys. Res. 97, 15 661-15 677 (1992).

${ }^{12}$ P. D. Thorne, P. J. Hardcastle, and R. L. Soulsby, "Analysis of acoustic measurements of suspended sediments," J. Geophys. Res. 98, 899-910 (1993).

${ }^{13}$ P. D. Thorne, P. J. Hardcastle, and A. Hogg, “'Observations of near-bed suspended sediment turbulence structures using multifrequency acoustic backscattering," in Coherent Flow Structures on Open Channels, edited by P. J. Ashworth, S. J. Bennett, J. L. Best, and S. J. McLelland (Wiley, London, 1996), pp. 343-358.

${ }^{14}$ J. F. Lynch, J. D. Irish, C. R. Sherwood, and Y. C. Agrawal, "Determining suspended sediment particle size information from acoustical and optical backscatter measurements," Continental Shelf Res. 14, 1139-1165 (1994).

${ }^{15}$ J. Sheng and A. E. Hay, "Sediment eddy diffusivities in the nearshore zone, from multifrequency acoustic backscatter," Continental Shelf Res. 15, 129-147 (1995).

${ }^{16}$ J. Sheng and A. E. Hay, "An examination of the spherical scatterer approximation in aqueous suspensions of sand,' J. Acoust. Soc. Am. 83, 598-610 (1988).

${ }^{17}$ A. M. Crawford and A. E. Hay, "Determining suspended sand size and concentration from multifrequency acoustic backscatter," J. Acoust. Soc. Am. 94, 3312-3324 (1993).

${ }^{18}$ P. D. Thorne, K. R. Waters, and T. J. Brudner, "Acoustic measurements of scattering by objects of irregular shape," J. Acoust. Soc. Am. 97, 242-251 (1995).

${ }^{19}$ C. M. Piaszczyk and J. M. Klosner, "Acoustic radiation from vibrating surfaces at characteristic frequencies," J. Acoust. Soc. Am. 75, 363-375 (1984).

${ }^{20} \mathrm{Z}$. Ruet, "On the boundary integral methods for the exterior acoustic problem," J. Sound Vib. 103, 297-298 (1985).

${ }^{21}$ M. Mamdi, Ph.D. thesis, University de technology de Compiegne, France, 1982.

${ }^{22}$ PAFEC VibroAcoustics, from Pafec Ltd, Strelley Hall, Nottingham N68 6PE, U.K.

${ }^{23}$ S. Sun, P. D. Thorne, I. K. Bjørnø, and T. Mazoyer, "'Observations of acoustic backscattering by elastic cubes," in The Proceedings of the 'Third European Conference on Underwater Acoustics' edited by J. S. Papadakis, and held at FORTH/IAMC on Crete, 24-28 June 1996, pp. 51-56.

${ }^{24}$ S. Sun, P. D. Thorne, and I. K. Bjørnd, "Using the specular component of sphere backscattering for broadband transceiver calibration," Acust. Acta. Acust. 82(4), 672-674 (1996).

${ }^{25}$ P. A. Chinnery, V. F. Humphrey, and J. Zhang, "Low frequency acoustic backscattering by a cube: Experimental measurements and theoretical prediction," J. Acoust. Soc. Am. 101, 2571-2582 (1997). 